\title{
Sexualidade e saúde sexual de adolescentes: interseção de demandas para o cuidado
}

\author{
Adolescent sexuality and sexual health: intersecting demands for care
}

Sexualidad y salud sexual de adolescentes: intersección de demandas para el cuidado

\author{
Rosália Teixeira Luz'; Edmeia de Almeida Cardoso Coelho"; Marizete Argolo Teixeirall'; Andiara Rodrigues Barros ${ }^{\text {IV }}$; \\ Maria de Fátima Alves Aguiar Carvalhov; Mariza Silva Almeida ${ }^{\text {II }}$
}

\begin{abstract}
RESUMO
Objetivo: conhecer as demandas para o cuidado no âmbito da sexualidade de adolescentes. Metodologia: estudo descritivo, abordagem qualitativa, tendo o gênero como categoria analítica. Realizado com 12 adolescentes de dois colégios públicos estaduais do interior da Bahia, em 2014. Foram utilizados oficinas de reflexão e questionário para coleta de dados e o tratamento se deu pela técnica de análise de discurso. Resultados: adolescentes consideram a sexualidade como dimensão humana que demanda atenção à saúde com necessidade de orientações e cuidado direcionados a vulnerabilidades e conhecimento do corpo. É também processo afetivosexual, com prazer compartilhado, caminho para superação de desigualdades de gênero. Conclusão: as demandas apresentadas revelam um grupo que defende uma sexualidade saudável e prazerosa, livre de tabus e preconceitos, mas carente de orientações. As demandas extrapolam o que está posto como prioridade para atenção à saúde e urge a aproximação escola e serviços de saúde para implementar políticas de formação e cuidado de adolescentes.
\end{abstract}

Descritores: Sexualidade; saúde sexual; saúde do adolescente; necessidades e demandas de serviços de saúde.

\section{ABSTRACT}

Objective: to examine demands for care in the field of adolescent sexuality. Methodology: deploying gender as an analytical category this qualitative, descriptive study was carried out in 2014 with 12 adolescents from two state public colleges in Bahia. Reflection workshops and a questionnaire were used for data collection and data were analysis by the discourse analysis technique. Results: adolescents consider sexuality to be a human dimension demanding health care, posing needs for guidance and care directed to vulnerabilities and body knowledge. It is also an affective and sexual process of shared pleasure, and a pathway to overcoming gender inequalities. Conclusion: the demands presented revealed a group that advocates healthy, pleasurable sexuality, free from taboos and prejudices, but lacking guidelines. The demands extend beyond what are considered health care priorities, and urge approximation between school and health services to implement adolescent education and care policies.

Descriptors: Sexuality; sexual health; adolescent health; health service needs and demands.

\section{RESUMEN}

Objetivo: conocer las necesidades para el cuidado en el área de la sexualidad de adolescentes. Metodología: estudio descriptivo, de enfoque cualitativo, siendo el género la categoría de análisis. Fue realizado junto a 12 adolescentes de dos colegios públicos estatales del interior de Bahía, en 2014. Se han utilizado talleres de reflexión y cuestionarios para la recolección de datos y el análisis se basó en la técnica de análisis del discurso. Resultados: adolescentes consideran la sexualidad como dimensión humana que demanda atención a la salud, con necesidad de orientaciones y cuidado dirigidos a las vulnerabilidades y el conocimiento del cuerpo. Es también un proceso afectivo-sexual, con placer compartido, un camino para la superación de desigualdades de género. Conclusión: las demandas presentadas revelan un grupo que aboga por una sexualidad saludable y placentera, sin tabús y prejuicios, pero con pocas orientaciones. Las demandas extrapolan lo que se muestra como prioridad para la atención a la salud y urge el acercamiento escuela y servicios de salud para poner en marcha políticas de formación y cuidado de los adolescentes.

Descriptores: Sexualidad; salud sexual; salud del adolescente; necesidades y demandas de servicios de salud.

\section{INTRODUÇÃO}

A adolescência constitui uma etapa de transição entre a infância e a idade adulta, marcada por transformações biológicas, psicológicas e sociais. Possui caráter sociocultural e é vivenciada de diferentes formas dependendo dos contextos em que adolescentes se inserem.

As mudanças corporais e a assunção de papéis sociais pelos adolescentes irão exigir um intenso trabalho de reelaboração psíquica. Um tempo ímpar na vida da pessoa, de mudanças geradoras de uma metamorfose afetiva, sexual e social ${ }^{1}$.

'Enfermeira. Doutora. Professora Adjunto, Universidade Estadual do Sudoeste da Bahia. Brasil. E-mail: rosluz@gmail.com "Enfermeira. Doutora. Professora Associada, Universidade Federal da Bahia. Brasil. E-mail: edmeiacoelho@gmail.com

I"Enfermeira. Doutora. Professora Titular, Universidade Estadual do Sudoeste da Bahia. Brasil. E-mail: marizete88@yahoo.com.br

IVEnfermeira. Mestre. Doutoranda, Hospital Universitário Professor Edgar Santos. Brasil. E-mail: andiarabarros@gmail.com

vEnfermeira. Doutora. Professora Adjunto, Universidade Federal do Vale do São Francisco. Brasil. E-mail: fatimaaguiar@hotmail.com.br

VIEnfermeira. Doutora. Professora Adjunta, Universidade Federal da Bahia. Brasil. E-mail: marizaal@yahoo.com.br

VIIFomento: Fundação de Amparo ao Pesquisador do Estado da Bahia. 
No tocante ao cuidado de adolescentes, o Programa Saúde do Adolescente, criado em 1989, foi a primeira iniciativa do Ministério da Saúde. Todavia, embora pautado no princípio da integralidade, perspectiva multidisciplinar e enfoque preventivo e educativo, não conseguiu ser implementado como proposto ${ }^{2}$. Outras estratégias e programas foram criados posteriormente em articulação com educação e assistência social, a exemplo da Política Nacional de Atenção à Saúde Integral de Adolescentes e Jovens, em 2004, e Programa Saúde na Escola, em 2007. Porém, têm sido implementados de modo pontual com ações desarticuladas ${ }^{3,4}$.

Diante dessa constatação, a questão desta pesquisa é: quais são as demandas de cuidado por objetivo: conhecer demandas para o cuidado no âmbito da sexualidade de adolescentes.

\section{REVISÃO DE LITERATURA}

$\mathrm{Na}$ atenção à adolescentes entende-se integralidade como a garantia de cuidado com escuta, acolhimento das demandas de saúde, procurando dar resposta às necessidades por meio da prevenção de agravos, promoção da saúde e recuperação de doenças ${ }^{5}$. Todavia, demandas de adolescentes, entendidas como expressão de necessidades de cuidado, são comumente difíceis de lidar segundo os padrões que se estabelecem nas instituições, sobretudo as que dizem respeito à sexualidade.

Sexualidade envolve comportamento e processos, incluindo os aspectos biopsicosociais, fisiológicos, culturais, políticos, espirituais e religiosos relacionados ao sexo e ao comportamento sexual. Desse modo, está ligada a uma dimensão da personalidade que é moldada por esses aspectos, estando muitas vezes reprimida e aprisionada no campo do desejo ${ }^{6}$. Ela remete a um prazer sensual, sedução, excitação, erotismo, orgasmo, conhecimento do próprio corpo e do corpo do outro?.

O processo de socialização vem acontecendo de forma rápida, especialmente nos países em desenvolvimento, com isso faz-se necessário que adolescentes encontrem pessoas capazes de lhes apoiar, pois a socialização é a maneira pelo qual o ser humano interioriza valores, crenças atitudes e normas de conduta que são próprias do seu grupo social, ou da sociedade, interferindo na atitude do adolescente em relação à sexualidade ${ }^{8}$.

Adolescentes necessitam ser orientados e estimulados, por meio de programas específicos. Para isso, é importante que sociedade e profissionais estejam treinados para aconselhamento sexual e para abordar a sexualidade. É preciso, contudo, que esses busquem fazer uma revisão de seus valores individuais para que não se perpetuem mitos, crenças e preconceitos e que a educação sexual formal não seja instrumento de repressão, mas uma contribuição positiva para o desenvolvimento integral do adolescente ${ }^{9}$.

\section{METOdOLOGIA}

Pesquisa descritiva de abordagem qualitativa, que tem o gênero como categoria analítica. No tocante à categoria analítica de gênero, essa foi construída a partir do reconhecimento de que papeis desempenhados por homens e mulheres são produtos de uma construção social e cultural, que veicula ideias, crenças, valores e símbolos e legitimam relações de poder, com privilegiamento do masculino ${ }^{10}$.

A pesquisa teve como participantes 12 adolescentes, estudantes de duas escolas públicas de uma cidade do interior da Bahia, sendo quatro do sexo masculino e oito do sexo feminino, dos quais oito tinham 18 anos de idade e quatro tinham 17 anos; oito cursavam o 3o ano do Ensino Médio e quatro relataram ser de religião evangélica, cinco são católicos e duas não possuíam religião.

As escolas foram escolhidas por constituir campo de prática para cursos de graduação em saúde de uma universidade do interior da Bahia, em julho de 2014. Os critérios de inclusão foram: estar matriculado nas escolas públicas selecionadas e ter entre 10 e 19 anos de idade, faixa etária considerada como adolescência pela Organização Mundial de Saúde (OMS)². O estudo respeitou as exigências da Resolução no 466/2012 do Conselho Nacional de Saúde, tendo sido seu projeto aprovado pelo Comitê de Ética em Pesquisa da Escola de Enfermagem da Universidade Federal da Bahia, sob parecer no 655.873, CAEE 27288414.2.0000.5531.

Os dados foram coletados por meio de duas oficinas de reflexão e um questionário. Oficinas surgem como uma proposta de articular subjetividade, racionalidade, experiência pessoal e conhecimento e, hoje, são utilizadas em diversas áreas como na psicologia, sociologia, enfermagem e em grupos autônomos de mulheres do movimento feminista, a partir da problematização ${ }^{11}$. No âmbito da pesquisa, a oficina se destaca pela articulação entre duas dimensões básicas e indissociáveis da construção de conhecimento, articulações teórico-metodológicas e implicações ético-políticas ${ }^{12}$.

As oficinas foram denominadas Adolescentes e necessidades de saúde. Foram constituídas pelas etapas de: integração, desenvolvimento do tema, incluindo a reflexão e a discussão sobre o tema; síntese e descontração. 
As oficinas foram desenvolvidas por duas pesquisadoras, ambas com experiência na estratégia metodológica. O processo foi fotografado, as falas foram gravadas e posteriormente, transcritas na íntegra para análise e discussão.

Dos diferentes temas abordados, os que diziam respeito à sexualidade necessitaram de aprofundamento, tendo em vista tratar de questões de foro íntimo e haver discussão mais restrita no grupo. Assim, foi aplicado questionário a nove adolescentes presentes em sala de aula nessa etapa da coleta de dados, na ausência das pesquisadoras e da professora responsável pela disciplina.

O material empírico foi transcrito na íntegra e tratado pela técnica de análise de discurso. O discurso é uma posição social, cuja formação ideológica é materializada na linguagem. Na análise do discurso, à medida que se estudam os elementos discursivos, vai sendo identificada a visão de mundo dos sujeitos inscritos nos discursos, em seguida, revelase o que a determinou. Foram seguidos os passos: leitura do texto buscando localizar as recorrências; depreensão de temas parciais; agrupamento de temas em bloco de significação; construção de categorias empíricas; análise do material articulado à literatura pertinente ${ }^{13}$.

As categorias empíricas que emergiram da análise, foram: saúde sexual e sexualidade como demandantes de informação e cuidado na adolescência; sexualidade como espaço de prazer compartilhado e de mistérios; e sexualidade como fonte de libido, prazer e fantasias sexuais.

Para garantir o anonimato das entrevistas, foram codificadas utilizando a letra inicial da palavra adolescente (A) seguida no numeral correspondente, e para facilitar a identificação do sexo, foi utilizada a letra $\mathrm{F}$ para designar o sexo feminino e a M para masculino.

\section{RESULTADOS E DISCUSSÃO}

Ao expressar seu olhar sobre a sexualidade, os discursos dos adolescentes revelaram interseção entre saúde sexual e sexualidade. Suas curiosidades e demandas compõem as categorias analisadas a seguir:

\section{Saúde sexual e sexualidade como demandantes de informação e cuidado na adolescência}

Os discursos dos adolescentes participantes desta pesquisa expressaram posições sociais em que a sexualidade apresenta intrínseca relação com a saúde sexual, conforme se lê a seguir:

Fase bastante conturbada, com modificações marcantes no corpo, onde algumas vezes é preciso de acompanhamento médico como, por exemplo, o ciclo menstrual. (A1, 18 anos, F)

Já tive e tenho necessidade em relação à ginecologista, rotina e dúvidas, nutricionista e fisioterapeuta. (A2, 18 anos, F)

Na adolescência, há bastante problemas e curiosidades e a questão da saúde está muito envolvida. É preciso ter acompanhamento sobre sexualidade (A3, 17 anos, $F)$.

Acho importante os assuntos que envolvem principalmente os cuidados com as relações sexuais; pois não é algo fácil, simples ou apenas prazerosa. Muitos pensam na sexualidade como apenas algo relacionado com o prazer e esquecem que é necessário cuidar da saúde. (A4, 18 anos, F)

Nos próximos depoimentos, ao serem questionados sobre curiosidades no âmbito da saúde que se relacionam com a sexualidade, enfatizam a necessidade de proteção pela camisinha nas relações sexuais, ao identificarem aspectos que expressam a relação sexualidade e saúde. Assim relatam:

Proteção sempre interfere na saúde, algumas pessoas não gostam de usar proteção e acabam com doenças. As pessoas se preocupam com gravidez, eu já penso que o pior é pegar uma doença. Por isso acho importante usar camisinha. Sexo sem proteção pode pegar AIDS ou uma gravidez. (A5, 17 anos, F)

O sexo com camisinha tem segurança. (A6, 18 anos, F)

Eu acho que camisinha é saúde, a gente tem que prevenir, pode até fazer com quem quiser, agora tem que prevenir. (A1, 18 anos, F)

Problemas relacionados à relação sexual, na maioria das vezes. As doenças como a [Síndrome da Imunodeficiência Adquirida] AIDS. (A7, 18 anos, M)

Sexo sem camisinhas, remédios para ajudar os homens, porque é perigoso, existem [doenças sexualmente transmissiveis] DST, existe o perigo de engravidar em um momento inesperado. (A8, 18 anos, $F$ )

Eu considero uma interferência na saúde a prática com muitas pessoas. Como todos sabem, as DST estão por toda parte. $(A 4,18$ anos, $F)$

Adolescentes que participaram do estudo expressam em seus discursos demandas no nível da prevenção, que requerem ações profissionais e remetem à necessidade de conhecimento do corpo. Na perspectiva da integralidade, que constitui eixo norteador da Política Nacional de Atenção à Saúde Integral de Adolescentes e Jovens e do Programa Saúde na Escola, urge que essa demanda seja atendida nos serviços de saúde e nas escolas por meio de processos 
educativos grupais em que adolescentes tenham voz. Nesses, trocas e aprendizado coletivo sobre corpo, sexualidade e saúde sexual abrem caminhos também para a superação de estereótipos de gênero que desigualam relações.

A saúde sexual representa a liberdade que mulheres e homens possuem de viver a sua sexualidade de forma consciente e sem risco de adquirir Infecções Sexualmente Transmissíveis (IST) e gestação não desejada. Para tanto, deve-se abordar a sexualidade humana de forma positiva e com respeito mútuo nas relações sexuais. A saúde sexual inclui o prazer e estimula a determinação, a comunicação e as relações interpessoais ${ }^{14}$.

Em estudo realizado no Amapá, foi constatado que o conhecimento de adolescentes sobre a sexualidade está relacionado ao ato sexual, à proteção contra gravidez, às doenças relacionadas ao sexo e à orientação sexual e evidencia a relação entre o ato sexual e os riscos de gravidez indesejada, o contágio por IST e a orientação sexual ${ }^{15}$.

Pesquisa recente mostrou que a iniciação sexual de adolescentes é precoce e mais de $90,0 \%$ dos participantes referiram conhecer ao menos uma $\mathrm{IST}^{16}$. Em outro estudo a maioria dos adolescentes já havia tido relação sexual, aproximadamente um terço não utilizou método contraceptivo na primeira relação e parcela significativa já esteve grávida ou a parceira engravidou, evidenciando a vulnerabilidade de jovens às IST e à gestação precoce devido a relações sexuais desprotegidas ${ }^{17}$.

No interior de Pernambuco, 282 adolescentes de escolas públicas municipais participaram de pesquisa que constatou a iniciação sexual de maneira precoce em ambos os sexos, de modo mais precoce entre adolescentes do sexo masculino, e o preservativo masculino como método anticoncepcional mais utilizado ${ }^{18}$.

Investigação com 29 estudantes de escola pública revelou divergência entre o que se estabelece nas leis e sua efetivação no cotidiano de adolescentes, com violações aos seus direitos sexuais, inexistência de educação voltada para as questões da sexualidade nas escolas e falta de serviços de saúde específicos de atenção a adolescentes, com consequências à saúde sexual ${ }^{19}$. Desse modo, sugere-se o desenvolvimento de estratégias dialógicas e de intervenções educativas emancipatórias, com troca de experiências entre profissionais da saúde, estudantes, escola e família para o exercício da cidadania sexual e redução de agravos relacionados à saúde sexual de adolescentes ${ }^{17-19}$.

A pertinente preocupação de adolescentes com o Vírus da Imunodeficiência Adquirida (HIV) AIDS é justificada pelo histórico dessa infecção. Entre 2003 e 2012, as maiores taxas de detecção de AIDS foram observadas entre pessoas com 30 a 49 anos. Contudo, observa-se uma tendência de queda desse grupo e uma leve estabilização entre as pessoas com 40 a 49 anos. Porém, nas taxas de detecção entre jovens de 15 a 24 anos e entre adultos com 50 anos ou mais existe tendência de aumento ${ }^{20}$.

Estudo realizado com 532 alunos de uma escola municipal do Rio Grande do Sul, que buscou avaliar o conhecimento e comportamento sexual de adolescentes sobre doenças sexualmente transmissíveis, mostrou que $89,2 \%$ das meninas e $90,3 \%$ dos meninos souberam definir adequadamente o conceito dessas doenças e para quase totalidade dos adolescentes o método preventivo mais eficaz foi o uso de preservativo. No entanto, $37,1 \%$ das meninas e $30,5 \%$ dos meninos referiram o uso de anticoncepcional como método preventivo para doenças sexualmente transmissíveis, evidenciando a importância da realização de ações educativas junto à escola sobre temas como sexualidade e saúde reprodutiva ${ }^{21}$.

As relações de gênero interferem tendo em vista a dificuldade de negociação do uso do preservativo, agravada na população feminina quando existe a subordinação econômica ao parceiro, pois geram novas dependências e a mulher tende a aceitar ter relações desprotegidas para não perdê-lo.

\section{Sexualidade como espaço de prazer compartilhado e de mistérios}

Adolescentes deste estudo traduzem sexualidade como um processo afetivo-sexual, que envolve satisfação em resposta ao desejo e à busca pelo prazer: Os depoimentos que seguem são representativos desta afirmação:

Quando se fala em prazer, logo se pensa em sexo. A sexualidade tem como resultado o prazer, a satisfação. (A9, 18 anos, F)

Desejo, vontade, prazer, conhecer outras pessoas, me relacionar. (A6, 18 anos, F)

Sexualidade é sentir prazer em fazer com quem ama. (A3, 17 anos, F)

Algo normal, que para muitas pessoas é algo polêmico, de constrangimento. Mas sim, é algo normal e todos vão fazer, porque faz parte e é bom. (A10, 17 anos, M)

Além de ser um assunto polêmico para muitas pessoas, no meu pensamento é a coisa mais normal do mundo, até porque, para eu nascer, houve isso. E eu também penso que esperar o tempo certo é sempre bom. (A8, 18 anos, F)

Já não me sinto mais surpreso com o assunto que é para mim uma coisa normal. (A11, 17 anos, M)

Um adolescente do presente estudo assume ser a beleza física feminina fonte de libido e de expectativa de prazer e de satisfação plena, alimentando fantasias. Com essa posição, ele reproduz uma cultura em que a beleza física é 
definidora da atração e das relações duradouras. Ao mesmo tempo, expressa dúvidas e curiosidades acerca da sexualidade, que oscilam entre a reafirmação do homem viril e a busca de superação da mentalidade machista. Pesquisa desenvolvida com jovens mostra resultado semelhante, eles se consideram sujeitos sexuais e de direitos, portanto tem direito ao exercício de suas sexualidades ${ }^{22}$.

Historicamente, a sexualidade esteve situada no campo do pecado e, sob dogmas da igreja católica foram impostas proibições ao seu exercício pleno, sobretudo às mulheres a quem foram associados todos os males advindos do desejo. Essas representações remontam à Idade Média, mas têm sua marca histórica no século XIX, em que a mulher passa a ser alguém a ser vigiada e controlada pela confissão, pelos homens ou pela família. O sexo passa a ter seu lugar no casamento e apenas para a procriação ${ }^{23}$.

No âmbito da vivência da sexualidade, curiosidade sobre bissexualidade e homossexualidade é demanda de adolescentes deste estudo. No período da adolescência nem sempre o comportamento sexual é uma característica fixa da sexualidade, podendo ser transitório ou mudado ao atingir a vida adulta. Desse modo, adolescentes com comportamento homo/bissexual possuem maior dificuldade nas relações com colegas e família devido aos tabus e preconceitos em torno do seu comportamento sexual ${ }^{24}$. No entanto, estudo realizado com 3.195 adolescentes de 10 estados brasileiros revelou que adolescentes com comportamento homo e bissexual tiveram mais facilidade em relatar vivências pessoais positivas e relacionamentos negativos do que heterossexuais, embora tenham falado menos sobre religiosidade ${ }^{25}$.

\section{Sexualidade como fonte de libido, prazer e fantasias sexuais}

Nos depoimentos seguintes, a sexualidade é considerada como fonte de libido, prazer e fantasias sexuais:

A primeira coisa que me vem à cabeça é uma companheira linda, que me faça sentir desejo, que me dê prazer, que satisfaça tanto os seus desejos quanto os meus. Tenho várias dúvidas, mas sempre levo para o mesmo propósito como ser um bom companheiro na cama. (A7, 18 anos, $M)$

Desejo, vontade, prazer e orgasmo múltiplos, seja com homem ou mulher. Sabendo que experiência não mata ninguém. $O$ uso de preservativos é necessário, já que nas festas perdemos a cabeça, hoje é muito fácil pegar doenças. Acho que sexo diariamente interfere muito, já que é fácil ficar cansado e fraco para outras atividades. (A11, 17 anos, M)

Curiosidade da prática sexual, tenho dúvidas sobre a união de casais do mesmo sexo, talvez possa ser uma relação amorosa ou conturbada e porque homens sentem tanto tesão. (A1, 18 anos, F)

Saber todas as precauções que devo tomar, saber o que fazer, como usar certos produtos que se usam. Sexo oral e anal, porque é um local que contém muitas bactérias. Como será, como eu vou me comportar, quais as sensações que eu vou sentir. (A12, 18 anos, $M)$

Este tema parece bem restrito, porém é muito amplo. Desperta principalmente curiosidade de aprofundar no assunto, mas me educo nos meus pensamentos, o desejo é algo incontrolável de não ser sentido, porém controlo, continuando assim uma curiosidade ampla. (A6, 18 anos, F)

Os discursos referentes à prática sexual como momento compartilhado superam a ideia de homem ativo e mulher passiva e revelam curiosidades de uma geração que busca mais livremente o direito ao prazer e ao orgasmo. Em simultaneidade, valorizam o sexo com proteção, sendo as mulheres as que mais se preocuparam com o cuidado à saúde, ainda quando suas falas expressaram o prazer como foco.

No entanto, por ser um tema ainda impregnado por preconceitos e tabus, muitos familiares, educadores e profissionais de saúde se mostram despreparados para discutir sexualidade, quando se fala é de forma tímida, enfocando mais os aspectos biológicos ligados à reprodução ${ }^{24}$. Um estudo transversal, realizado em 147 unidades ambulatoriais do Rio de Janeiro, mostrou que em apenas em 12,9\% das unidades são realizadas atividades educativas direcionadas a adolescentes e que menos de $1 / 3$ dos médicos estavam capacitados para lidar com a saúde de adolescentes. Além de que em mais de $90 \%$ das unidades, adolescentes são atendidos junto a população adulta; em mais de $10 \%$ dos serviços, a assistência só é dada na presença do responsável, evidenciando que a estrutura do atendimento e os recursos humanos não acolhem as demandas do público adolescente, apesar da larga distribuição geográfica das unidades de saúde ${ }^{26}$.

A educação em saúde que segue o modelo dialógico busca respeitar as diferentes visões de mundo e de significados entre adolescentes e adultos, de modo a favorecer as relações intergeracionais ${ }^{22}$, e, desse modo, abre caminhos para que pessoas se tornem autônomas e corresponsáveis no cuidado e na promoção da sua saúde. Para tanto, profissionais de saúde devem ser facilitadores de todo processo educativo, para que o cuidado se desenvolva sob o eixo da integralidade e da busca da autonomia do adolescente.

Tendo em vista a transversalidade de gênero, as políticas públicas devem ser permeadas por essa perspectiva, o que implica reconhecer e incorporar a construção social e cultural dos papeis naturalizados para mulheres e homens, 
bem como problematizar a realidade no caminho da superação de desigualdades nas relações e do empoderamento das mulheres.

\section{CONCLUSÃO}

Os adolescentes que participaram desta pesquisa consideram a sexualidade como dimensão que demanda atenção à saúde, e ressaltaram a necessidade de orientações preventivas, acompanhamento profissional e conhecimento das alterações biológicas do corpo. Em nível de igual importância, valorizam sexo com proteção para que preservem a saúde, enfatizando o uso da camisinha nas relações sexuais, para evitar IST/HIV/AIDS e/ou uma gravidez precoce.

A sexualidade é considerada por eles um processo afetivo-sexual, que envolve satisfação em resposta ao desejo, e eles superam a ideia de homem ativo e mulher passiva, representando uma geração mais livre para a busca do prazer compartilhado. É também lugar de mistério, curiosidades e dúvidas e o sexo é considerado algo que faz parte da vida, mas ainda um tema que causa constrangimento e polêmica entre as pessoas. Sobretudo entre as adolescentes, foram valorizados o envolvimento afetivo e a preocupação com o uso da camisinha.

Embora o estudo apresente como limitação ter sido desenvolvido em apenas duas escolas públicas do município, ele revela que os adolescentes entrevistados apresentam um olhar diferenciado para as relações e para a sexualidade, de modo que o sistema de saúde, conforme organizado na atualidade, não tem respostas para suas demandas e necessidades de saúde. Isso porque extrapolam o que está posto como prioridades pelos serviços, em que ações dirigidas à prevenção, circunscritas ao modelo clínico lideram as ofertas para o grupo.

\section{REFERÊNCIAS}

1. Costa SMBC, Machado MTC. Body and body image among adolescents: outlook from the comprehensive healthcare standpoint. Adolesc saúde. 2014 [cited 2018 Jan 27]; 11(2):19-24. Available from: http://www.adolescenciaesaude.com/detalhe_artigo.asp?id=441

2. Ayres JRCM, Carvalho YM, Nasser MA, Saltão RM, Mendes VM. Ways of comprehensiveness: adolescents and young adults in Primary Healthcare. Interface[Online]. 2012 [cited 2018 Jan 27]; 16(40):67-82. Doi: http://dx.doi.org/10.1590/S141432832012005000021

3. Melo MCP, Coelho EAC, Galvão MTG, Nascimento ER. Comprehensiveness and gender as theoretical basis for health care of pregnant adolescents [Online]. 2013 [cited 2018 Jan 27]; 17(3):736-40. Doi: http://www.dx.doi.org/10.5935/14152762.20130054

4. Leite $\mathrm{V}$. The adolescent sexuality from the perspective of policymakers: reflecting the ideology of adolescents as subjects of rights. Psicol. clin. [Online]. 2012 [cited 2018 Jan 27]; 24(1):89-103. Doi: http://dx.doi.org/10.1590/S0103-56652012000100007

5. Ministério da Saúde (Br). Secretaria Executiva. Coordenação da Saúde da Criança e do Adolescente. Programa Saúde do Adolescente. Bases Programáticas. Brasília (DF): Editora MS; 1996.

6. Nwagu EN. Alcohol and drug usage; and adolescents' sexual behaviour in Nigeria. Health Promot Int.[Online]. 2016 [cited 2018 Jan 27]; 31(2):405-13. Doi: http://dx.doi.org/10.1093/heapro/dav001

7. Greenberg JS, Bruess CE, Oswalt SB. Exploring the dimensions of human sexuality. Burlington: Jones \& Bartlett Learning; 2014.

8. Vassilikou K, loannidi KE. Sex education and sex behaviour in Greek adolescents: a research review. Soc. Cohesion \& Develop [Online]. 2014 [cited 2018 Jan 27]; 9(2):143-54. Doi: http://dx.doi.org/10.12681/scad.8912

9. Silva IR, Leite JL, Lins SMSB, Silva TP, Santos MJC. Orders and disorders: the complexity of adolescence and sexual health contributions to nursing. Rev. enferm. UERJ.[Online]. 2016 [cited 2018 Jan 27]; 24(2):e14569. Doi: http://dx.doi.org/10.12957/reuerj.2016.14569

10. Scott JW. Os usos e abusos do gênero. Projeto História. 2012 ; 45(1): 327-51.

11. Santos CC, Alves CN, Barreto CN, Wilhelm LA, Cremonese L, Ressel LB. Vivenciando oficinas lúdico-pedagógicas: uma nova experiência de pensar e fazer a enfermagem com adolescentes. Adolesc. Saúde [Internet]. 2014 cited 2018 Jan 27; $11(1): 63-7$.

12. Spink MJ, Menegon VM, Medrado B. Using workshops as a research strategy: theoretical and methodological articulations and ethical-political applications. Psicol. soc. [Online]. 2014 [cited 2018 Jan 27]; 26(1):32-43. Doi: http://dx.doi.org/10.1590/S010271822014000100005.

13. Fiorin JL. Linguagem e ideologia. São Paulo: Ática; 2011.

14. Kar SK, Choudhury A, Singh AP. Understanding normal development of adolescent sexuality: a bumpy ride. J. Hum. Reprod. Sci. [Online]. 2015 [cited 2018 Jan 27]; 8(2):70-4. Doi: http://dx.doi.org/10.4103/0974-1208.158594

15. Ferreira EA, Alves VH, Pereira AV, Rodrigues DP, Paiva ED, Santos IMM. Adolescents in the school environment and knowledge of sexual and reproductive health. Cogitare enferm. Rev. [Online]. 2018 [cited 2018 Dec 20]; (23)2:e55851. Doi: http://dx.doi.org/10.5380/ce.v23i2.55851

16. Santos MKLF, Queiroz LA, Saltirio ILS, Rios JSC, Pinho HMLS, Silva KWL et al. Sex education strategies from the perception of students at a public school. Arq. Med. Hosp. Fac. Cienc. Med. Santa Casa São Paulo [Online]. 2018 [cited 2018 Jan 27]; 63(2):905. Doi: https://doi.org/10.26432/1809-3019.2018.63.2.90

17. Maciel KMN, Andrade MS, Cruz LZ, Fraga CDS, Paixão GPN, Souza RS. Characteristics of teenage sexual behavior. Rev. enferm. UERJ. [Online]. 2017 [cited 2018 Jan 27]; 25:e23496. DOI: http://dx.doi.org/10.12957/reuerj.2017.23496 
18. Lins LS, Silva LAM, Santos RG, Morais TBD, Beltrão TA, Castro JFL. Analysis of the sexual behavior of adolescents. Rev. bras. promoç. saúde. [Online]. 2017 [cited 2018 Jan 27]; 30(1):47-56. DOI: http://dx.doi.org/10.5020/18061230.2017.p47

19. Campos HM, Paiva CGA, Mourthé ICA, Ferreira YF, Assis MCD, Fonseca MC. Dialogues with adolescents on sexual rights in public school: emancipatory educational interventions Pesqui. prát. psicossoc. 2018; 13(3):1-16. Available from: http://pepsic.bvsalud.org/scielo.php?script=sci_abstract\&pid=S1809-89082018000300010\&lng=pt\&nrm=iso\&tlng=en

20. Ministério da Saúde (Br). Secretaria de Vigilância em Saúde. Departamento de DST, Aids e Hepatites Virais. Recomendações para a atenção integral a adolescentes e jovens vivendo com HIV/Aids. Brasília (DF): Editora MS; 2013.

21. Genz N, Meincke SMK, Carret MLV, Corrêa ACL, Alves CN. Sexually transmitted diseases: knowledge and sexual behavior of adolescents. Texto \& contexto enferm. [Online]. 2017 [cited 2018 Jan 27]; 26(2). Doi: http://dx.doi.org/10.1590/010407072017005100015.

22. Silva CG, Borba PLO. Encounters with the difference at health education: youth, sexualities, and genders at school. Saúde soc. [Online]. 2018 [cited 2018 Jan 27]; 27(4):1134-46. Doi: https://doi.org/10.1590/S0104-12902018170274

23. Duarte A, César MRA. Is it useless to resist against the sexual apparatus? Foucault and Butler on bodies and pleasures. Rev. Filos. Aurora. [Online]. 2016 [cited 2018 Jan 27]; 28(45);949-67. Doi: http://dx.doi.org/10.7213/1980-5934.28.045.DS11

24. Saito MI, Silva LEV. Adolescência: prevenção e risco. Rio de Janeiro: Atheneu; 2014.

25. Assis SG, Gomes R, Pires TO. Adolescence, sexual behavior and risk factors to health. Rev. saúde pública. [Online]. 2014 [cited 2018 Jan 27]; 48(1):43-51. Doi: http://dx.doi.org/10.1590/S0034-8910.2014048004638.

26. Taquette SR, Monteiro DLM, Rodrigues NCP, Rozenberg RMDCS, Rodrigues AO, Menezes DCS et al. Sexual and reproductive health among young people, Rio de Janeiro, Brazil. Ciênc. saúde coletiva. [Online]. 2017 [cited 2018 Jan 27]; 22(6):1923-32. Doi: http://dx.doi.org/10.1590/1413-81232017226.22642016 